\title{
AS BIBLIOTECAS COMO FACILITADORAS NO ACESSO À INFORMAÇÃO POR USUÁRIOS COM DEFICIÊNCIA VISUAL
}

LIBRARIES AS FACILITATORS IN ACCESS TO INFORMATION BY VISUALLY IMPAIRED USERS

LAS BIBLIOTECAS COMO FACILITADORAS EN EL ACCESO A LA INFORMACIÓN POR USUARIOS CON DEFICIENCIA VISUAL

${ }^{1}$ Tania Milca Malheiros, ${ }^{1}$ Murilo Bastos da Cunha

1Universidade de Brasília

Correspondência

'Tania Milca Malheiros

Universidade de Brasília

Brasília, DF

Email: tania@bce.unb.br

ORCID: https://orcid.org/0000-0002-1642-6298

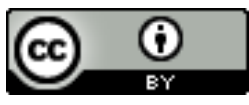

Submetido em: 07/09/2017

Aceito em: 13/10/2017

Publicado em: 24/10/2017

JITA: CC. User categories: children, young people, social groups. 
RESUMO: Este artigo trata do papel das bibliotecas como colaboradoras no processo de aquisição da informação pelos usuários com deficiência visual. Ele tem como objetivo mostrar a importância desse papel no processo de inclusão social desses usuários e a necessidade de se discutir esta temática. Como ponto fundamental para sua inclusão encontram-se a produção e oferta de materiais alternativos. São citadas as barreiras enfrentadas no processo de aquisição, como a falta de materiais adaptados e o custo de produção. Também é apresentado um histórico da aquisição de informações desses usuários e de sua inclusão em bibliotecas brasileiras e no exterior. O método de pesquisa utilizado foi a revisão de literatura, tendo sido consultadas as bases de dadosLibrary and Information Science Abstracts (LISA), Library \& Information Science and TechnologyAbstracts (LISTA), Information Science and Technology Abstracts (ISTA), a Base de dados de Periódicos em Ciência da Informação (BRAPCI), Google e Google Acadêmico.Como conclusão, apontamos a cooperação entre os serviços de bibliotecas como solução para essa questão.

PALAVRAS-CHAVE: Biblioteca. Deficiente visual. Inclusão social. Produto de informação. Serviço de informação.

ABSTRACT: This article deals with the role of libraries as collaborators in the process of information acquisition by visually impaired users. It aims to show the importance of this role in the process of social inclusion of these users and the need to discuss this issue. As a fundamental point for its inclusion are the production and supply of alternative materials. The barriers faced in the process of information acquisition are cited, such as the lack of adapted materials and the cost of production. It also presents a history of the acquisition of information from these users and their inclusion in Brazilian and foreign libraries. The research method used was the literature review having been consulted the databaseLibrary and Information Science Abstracts (LISA), Library \& Information Science and TechnologyAbstracts (LISTA), Information Science and Technology Abstracts (ISTA), Base de dados de Periódicos em Ciência da Informação (BRAPCI), Google and Google Scholar.As a conclusion, we point out the cooperation between library services as a solution to this issue.

KEYWORDS: Information products. Information services. Libraries. Reference service. Social inclusion. Visually impaired users.

RESUMEN: Este artículo trata del papel de las bibliotecas como colaboradoras en el proceso de la recuperación de la información por los usuarios con deficiencia visual. Pretende mostrar la importancia de esta función en el proceso de inclusión social de los usuarios y la necesidad de discutir el tema en la búsqueda de soluciones. Como punto fundamental para su inclusión se encuentran la producción y oferta de materiales alternativos. Se citan las barreras enfrentadas en el proceso de adquisición, como la falta de materiales adaptados y el costo de la producción. También se presenta un historial de la recuperación de la información de esos usuarios y de su inclusión en bibliotecas brasileñas y en el exterior. El método de investigación utilizado fue la revisión de la literatura,habiendo sido consultadas las bases de datos, Library and Information ScienceAbstracts (LISA), Library \& Information Science and TechnologyAbstracts(LISTA), Information Science and TechnologyAbstracts (ISTA), Base de dados de Periódicos em Ciência da Informação (BRAPCI), Google y Google Scholar. Como conclusión, señalamos la cooperación entre los servicios de las bibliotecas como solución a esta cuestión.

PAlabras Clave: Biblioteca. Deficiente visual. Inclusión social. Producto de información. Servicio de información. 


\section{INTRODUÇÃO}

O impacto que a tecnologia da informação (TI) tem tido na vida das pessoas é fato comprovado por vários autores na literatura. O processo de comunicação entre as pessoas, até o advento da internet, ocorria de forma mais simples e o número de informações disponíveis era bem menor. Com a TI, temos cada vez mais acesso a um número maior de informações por meio de aparelhos e dispositivos com crescente grau de sofisticação. Aqui estamos falando de usuários que têm a livre escolha das informações que querem consumir. É claro que esse acesso passa pelas barreiras enfrentadas por cada consumidor, como, por exemplo, os recursos financeiros.

Trazendo a discussão para as pessoas com deficiência, em particular as que têm deficiência visual, essa realidade se transforma. Se, por um lado, a TI trouxe para as suas vidas o acesso a uma gama de informações, por outro, a falta de acessibilidade digital funciona como uma barreira a determinados tipos de informação. Para esses usuários não existe escolha, o seu consumo de informações está condicionado àquelas adaptadas às suas limitações, e, infelizmente, essas informações não são muitas.

Segundo Kavanagh; Skold (2009) e a World Blind Union (2013), de 5\% a 7\% do que é publicado pelo mercado editorial dos países desenvolvidos e menos de $1 \%$ nos países em desenvolvimento é disponibilizado em formato adaptado.

Essa situação de pouca disponibilização de materiais adaptados iniciou-se com as publicações em meio impresso e se agravou com o meio digital pela velocidade com que as informações são difundidas, visto que o processo de adaptação é lento e oneroso.

Brazier (2011) afirma que, no Reino Unido, existem 1,8 milhões de cegos ou pessoas com deficiência visual, a maioria com mais de 65 anos e muitos com problemas de saúde e mobilidade. Para essas pessoas, encontrar o suficiente para ler é um desafio porque menos de $5 \%$ dos livros são transcritos em formatos acessíveis a eles, como áudio, livros ampliados ou em braile. Vale ressaltar que os formatos em áudio e em braile necessitam de algum tipo de adaptação para se tornarem acessíveis (descrição de imagens, formatação, etc.).

Para amenizar esse cenário, é fundamental a ação das bibliotecas na preparação e no fornecimento de materiais alternativos. Em consequência dessa situação e para melhorar o acesso à biblioteca e aos serviços de informação para as pessoas com deficiência visual, a instituição Share the Vision, no Reino Unido uniu-se à Sociedade de Bibliotecários-Chefe para lançar uma iniciativa experimental na região Nordeste desse país. Esse projeto teve como objetivos estender a oferta das bibliotecas que disponibilizavam produtos e serviços de informação (PSI) dessa região para usuários de outras bibliotecas. Além disso, o projeto visou fazer com que essa oferta chegasse a esses usuários de forma eficaz, promovendo a integração dos PSI e desenvolvendo modelos sustentáveis na prestação dos serviços.

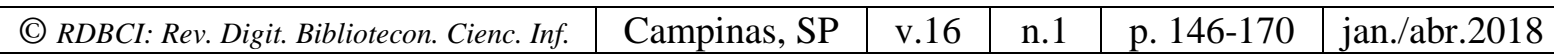


Uma das barreiras a ser enfrentada por esses materiais é o seu alto custo de produção. Vitzansky (1994) afirma que esses dependem de fundos públicos ou privados. O mercado editorial não se interessa por essa produção porque é pouco atrativa devido a seu caráter restrito, um pequeno nicho de mercado.

Alguns autores como Rabelo (1989), Griebel (2000), Bernardi (2004), Kanavanagh e Skold $(2005,2009)$ afirmam que uma forma de se melhorar essa situação é a cooperação entre os serviços de bibliotecas, o que pode diminuir os custos e eliminar a duplicação de trabalho.

As bibliotecas públicas neozelandesas que disponibilizam livros ampliados e em áudio tentaram por alguns anos a cooperação entre a Biblioteca da Fundação Real Nova Zelândia para Cegos (RNFZB) e a National Library of New Zealand's Print Disabilities Collection, para produção e empréstimo de audiolivros para as bibliotecas públicas. Entretanto, como o custo de produção ficou muito alto para um mercado muito pequeno, a parceria não se firmou. Por outro lado, um exemplo de cooperação bem-sucedido é o das bibliotecas americanas, que dispõem de uma rede que atende as necessidades de informação dos usuários, por meio do empréstimo e de um catálogo unificado (WILSON, 2003).

Com a chegada da nova realidade, a informação em meio digital, a adoção de padrões para os serviços bibliotecários foi necessária para que se garantisse o intercâmbio de dados, pela questão da interoperabilidade dos sistemas de bibliotecas e do apoio às redes nacional e internacional de bibliotecas.

O lançamento de livros em formato Daisy pela International Federation of Library Association and Institutions(IFLA) / Library for the Blind Section (LBS), em 1994, serviu como estímulo à adoção dos padrões que facilitam a troca de informações e a redução de custos no oferecimento de livros acessíveis (KAVANAGH; SKOLD, 2009, p. 12). Esse fato é de grande importância levando em consideração a realidade dura e difícil do acesso à informação pelos deficientes visuais, sem o poder de escolha do que vai poder ler.

A partir daí aIFLA também publicou outras diretrizes para o desenvolvimento de bibliotecas para as pessoas com deficiência visual ${ }^{1}$, que são de extrema importância para que os profissionais da informação tenham parâmetros na criação de bibliotecas ou serviços de bibliotecas para esses usuários. Com essa ação, a IFLA estimulou a redução de custos e a criação de novas bibliotecas.

Essas diretrizes abordam pontos fundamentais para a estruturação e criação dessas bibliotecas, entre eles, a necessidade de elaboração de políticas de desenvolvimento de

\footnotetext{
1،Para os propósitos destas diretrizes, pessoas incapazes de utilizar material impresso serão aquelas incapazes de utilizar material impresso devido à cegueira, baixa visão, uma deficiência de aprendizagem ou uma deficiência física" (KAVANAGH; SKOLD, 2009, p. 9).
}

\begin{tabular}{l|l|l|l|l} 
v.16 & n.1 & p. $146-170$ & jan./abr.2018
\end{tabular}


coleções, de utilização dos padrões técnicos, de criação de uma legislação específica, de produção de materiais alternativos e de cooperação e criação de redes. Têm como objetivo principal:

Prover bibliotecas, governos e outros mantenedores com uma estrutura para o desenvolvimento de serviços de bibliotecas para pessoas incapazes de utilizar material impresso. Quando apropriadas, estas diretrizes são confirmadas com exemplos de diversos países ao redor do mundo (KAVANAGH; SKOLD, 2009, p. 12).

Todas essas iniciativas e propostas são fundamentais para trazer o assunto à tona, gerando discussões que podem buscar soluções, pois sabemos que, mesmo nos países desenvolvidos, a oferta de materiais acessíveis é de 5 a $7 \%$ do total dos materiais publicados em seus países (idem, 2009, WORLD BLIND UNION, 2013).

\section{METODOLOGIA}

O método de pesquisa utilizado foi a revisão de literatura, tendo sido consultadas as bases de dadosLibrary and Information Science Abstracts (LISA), Library \& Information Science and TechnologyAbstracts (LISTA), Information Science and Technology Abstracts (ISTA), aBase de dados de Periódicos em Ciência da Informação (BRAPCI),o Google e o Google Acadêmico.

As estratégias de busca utilizadas e os resultados dos levantamentos podem ser visualizados nos quadros 1 e 2 .

QUADRO 1: Termos utilizados nos levantamentos de abril de 2014 e abril e outubro de 2016, e 2017

\begin{tabular}{|c|c|c|c|}
\hline \multicolumn{2}{|c|}{$\begin{array}{l}\text { Termos utilizados no Google/ } \\
\text { Google acadêmico }\end{array}$} & \multicolumn{2}{|c|}{ Termos utilizados nas bases de dados } \\
\hline $\begin{array}{l}\text { Termos em } \\
\text { português }\end{array}$ & $\begin{array}{l}\text { Termos em } \\
\text { inglês }\end{array}$ & $\begin{array}{l}\text { Termos em } \\
\text { português }\end{array}$ & Termos em inglês \\
\hline $\begin{array}{l}\text { Definição de } \\
\text { produtos e } \\
\text { serviços } \\
\text { informativos }\end{array}$ & $\begin{array}{l}\text { products and } \\
\text { services for } \\
\text { blind peoples }\end{array}$ & $\begin{array}{l}\text { Deficiente } \\
\text { visual }\end{array}$ & \multirow{3}{*}{$\begin{array}{l}\text { SU ("PEOPLE with disabilities -- Services for" } \\
\text { AND Su (visual OR blind) OR SU ("BLIND -- } \\
\text { Services for") }\end{array}$} \\
\hline $\begin{array}{l}\text { Unidades de } \\
\text { informação }\end{array}$ & Library & $\begin{array}{l}\text { Deficientes } \\
\text { visuais }\end{array}$ & \\
\hline $\begin{array}{l}\text { Usuários com } \\
\text { deficiência } \\
\text { visual }\end{array}$ & libraries & Cego & \\
\hline
\end{tabular}

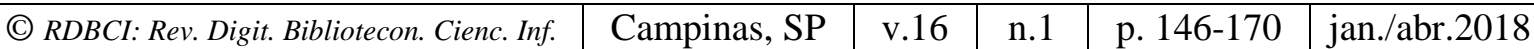




\begin{tabular}{|c|c|c|c|}
\hline $\begin{array}{l}\text { Produtos e } \\
\text { serviços } \\
\text { informativos } \\
\text { para pessoas } \\
\text { com deficiência } \\
\text { visual e as } \\
\text { sociedades } \\
\text { inclusivas }\end{array}$ & \multirow[t]{2}{*}{$\begin{array}{l}\text { marketing of } \\
\text { library and } \\
\text { information } \\
\text { products and } \\
\text { services }\end{array}$} & Biblioteca & \multirow[t]{2}{*}{$\begin{array}{l}\text { SU BLIND* AND SU (SERVICE* OR } \\
\text { PRODUCT*) } \\
\text { blind and partially sighted" } \\
\text { "blind and partially-sighted" } \\
\text { "blind and visually handicapped" } \\
\text { "blind and partially users" } \\
\text { "blind and partially sighted people"; Service (s); } \\
\text { Product (s) } \\
\text { LIBRARIES \& people with visual disabilities }\end{array}$} \\
\hline Cegos & & $\begin{array}{l}\text { Ciência da } \\
\text { informação }\end{array}$ & \\
\hline $\begin{array}{l}\text { Produtos de } \\
\text { informação }\end{array}$ & $\begin{array}{l}\text { "products and } \\
\text { services for } \\
\text { blind } \\
\text { peoples". }\end{array}$ & \multirow{3}{*}{ Serviços } & \multirow{3}{*}{$\begin{array}{l}\text { su(Exact("blind and partially sighted" OR "blind } \\
\text { and partially-sighted" OR "blind and visually } \\
\text { handicapped" OR "blind and partially users" OR } \\
\text { "blindness" OR "blind and partially sighted } \\
\text { people") OR BLIND) AND su ((SERVICE* OR } \\
\text { PRODUCT*)) }\end{array}$} \\
\hline $\begin{array}{l}\text { Deficientes } \\
\text { visuais }\end{array}$ & & & \\
\hline $\begin{array}{l}\text { Serviços de } \\
\text { informação }\end{array}$ & & & \\
\hline $\begin{array}{l}\text { Pessoas com } \\
\text { deficiência } \\
\text { visual }\end{array}$ & & \multirow{8}{*}{$\begin{array}{l}\text { "ciência da } \\
\text { informação" } \\
\text { OR } \\
\text { "biblioteca" } \\
\text { OR } \\
\text { "bibliotecon } \\
\text { omia" E } \\
\text { Todos os } \\
\text { campos: } \\
\text { cego OR } \\
\text { cegos OR } \\
\text { "deficiente } \\
\text { visual" OR } \\
\text { "deficiente } \\
\text { visuais" OR } \\
\text { "deficiencia } \\
\text { visual") } \\
\text { (Título: } \\
\text { "Ciência da } \\
\text { informação" } \\
\text { OR } \\
\text { "biblioteca" } \\
\text { OR } \\
\text { "bibliotecon } \\
\text { omia" E } \\
\text { Todos os } \\
\text { campos: } \\
\text { cego OR }\end{array}$} & \multirow{8}{*}{$\begin{array}{l}\text { ((DE "LIBRARIES \& the blind" OR DE } \\
\text { "LIBRARIES \& people with visual disabilities" } \\
\text { OR DE "PEOPLE with visual disabilities") OR } \\
\text { (DE "LIBRARIES \& the blind") ) AND SU ( } \\
\text { service* or product*) }\end{array}$} \\
\hline Marketing & & & \\
\hline $\begin{array}{l}\text { Tecnologia } \\
\text { assistiva }\end{array}$ & & & \\
\hline $\begin{array}{l}\text { Acessibilidade } \\
\text { digital }\end{array}$ & & & \\
\hline $\begin{array}{l}\text { "Bibliotecas } \\
\text { digitais } \\
\text { acessíveis } \\
\text { destinadas a } \\
\text { deficientes } \\
\text { visuais no } \\
\text { Brasil" }\end{array}$ & & & \\
\hline $\begin{array}{l}\text { "Bibliotecas } \\
\text { digitais para } \\
\text { deficientes } \\
\text { visuais no } \\
\text { Brasil" }\end{array}$ & & & \\
\hline $\begin{array}{l}\text { Usuários com } \\
\text { baixa visão }\end{array}$ & & & \\
\hline $\begin{array}{l}\text { Usuários com } \\
\text { deficiência }\end{array}$ & & & \\
\hline
\end{tabular}

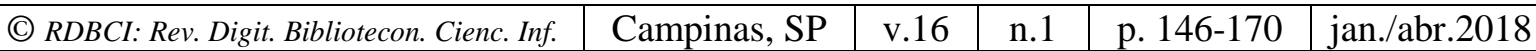




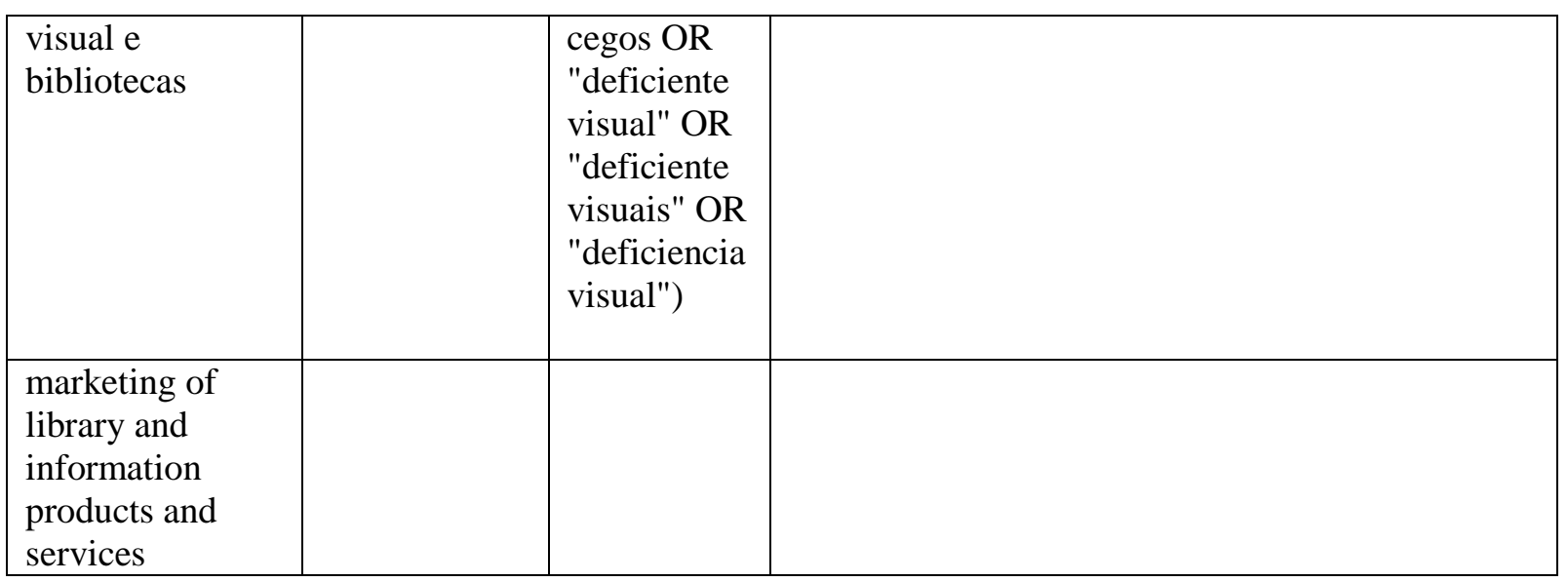

Fonte: Os autores.

QUADRO 2: Documentos recuperados e relevantes resultantes dos levantamentos realizados em 2014, 2016 e 2017

\begin{tabular}{|c|c|c|c|c|c|c|c|c|}
\hline \multirow[t]{2}{*}{ Fontes } & \multicolumn{2}{|c|}{$\begin{array}{c}1^{\circ} \text { Levantamento } \\
2014\end{array}$} & \multicolumn{2}{|c|}{$\begin{array}{c}2^{\mathbf{o}} \\
\text { Levantamento } \\
\text { Abril } 2016\end{array}$} & \multicolumn{2}{|c|}{$\begin{array}{c}3^{\circ} \\
\text { Levantamento } \\
\text { Outubro } 2016\end{array}$} & \multicolumn{2}{|c|}{$\begin{array}{c}4^{\circ} \\
\text { Levantamento } \\
\text { Agosto } 2017\end{array}$} \\
\hline & $\begin{array}{c}\text { Itens } \\
\text { recuperados } \\
\text { (IRec) }\end{array}$ & $\begin{array}{c}\text { Itens } \\
\text { relevantes } \\
\text { (IRel) }\end{array}$ & IRec & IRel & IRec & IRel & IRec & IRel \\
\hline LISA & 192 & 51 & 311 & 55 & - & 6 & 624 & 69 \\
\hline LISTA & 127 & 30 & 263 & 40 & - & - & 314 & 9 \\
\hline ISTA & 2 & 2 & 18 & 6 & - & - & 25 & 7 \\
\hline BRAPCI & 18 & 15 & 112 & $14+$ & - & - & 105 & 20 \\
\hline BDTD & 5 & - & 43 & 7 & - & - & - & - \\
\hline $\begin{array}{c}\text { Repositório } \\
\text { Instituciona } \\
1 \\
\text { UnB }\end{array}$ & 3 & 1 & 2 & - & - & - & - & - \\
\hline Google & 30 & 13 & 14 & 14 & - & - & - & - \\
\hline Outros & 9 & 6 & 1 & - & - & - & - & - \\
\hline
\end{tabular}

Fonte: os autores.

Com o resultado dos levantamentos foram selecionados os materiais relevantes para a fundamentação teórica deste artigo. 


\section{A CIÊNCIA dA INFORMAÇÃO E O PROCESSO dE INCLUSÃO SOCIAL}

Um dos meios de inclusão social é o acesso à informação. Como definir a informação e como dimensionar o seu valor? Segundo Fullmer e Majumder (1991, p. 17):

Informação/conhecimento é poder. A habilidade para obter e usar informações sobre algum assunto dá à pessoa a oportunidade de escolher um caminho de muitas alternativas, em vez de se limitar a algumas opções talvez não desejadas e inviáveis.

Sabemos da importância da informação para o desenvolvimento do ser humano em toda a sua dimensão. Principalmente no caso das pessoas com deficiência visual, pelas dificuldades enfrentadas no seu acesso e aquisição, acessar informações é essencial para sua socialização e formação educacional, em especial quando se trata da informação em meio digital, que trouxe para esses cidadãos mais autonomia.

Gerber (2003) ressalta a importância do uso do computador e do acesso à internet na vida das pessoas com deficiência visual, como melhoria educacional, oportunidade de emprego, aumento da socialização pelas redes sociais (por e-mail e grupos online) e da independência (com acesso pessoal à informação).

Na literatura da Ciência da Informação, são várias as definições de "informação". Le Coadic (2004, p. 4) aponta que "a informação é um conhecimento inscrito (registrado) em forma escrita (impressa ou digital), oral ou audiovisual, em um suporte”. Beal (2004, p. 12) define informação como "dados dotados de relevância e propósito"; já Buckland (1991, p. 353) conceitua a informação de três formas:

(a) a informação é um processo - como uma referência à sua propriedade de informar ou comunicar; (b) é um conhecimento - quando se refere ao que é passado na ação de informar ou comunicar; (c) é uma matéria, quando é vinculada aos dados e, consequentemente, ao suporte físico, onde esses dados são registrados.

Seguindo as definições dos autores acima citados, podemos concluir que um conjunto de dados organizados pode ser considerado informação, a qual em um contexto pode ser considerada como conhecimento que vai ser comunicado ou transmitido a alguém. Esse conhecimento deve ser organizado e disponibilizado para atender a necessidade de informação (NI) dos diversos usuários, seja em sua vida profissional ou pessoal. Hoje, a ciência que tem como ocupação principal o tratamento e fornecimento da informação, que resulta na organização desses conhecimentos, é a Ciência da Informação.

Segundo Le Coadic (2004, p. 19), é "uma ciência social" que tem como preocupação o acesso à informação pelos diversos usuários, e a área que cuida do mapeamento de suas NI é a área de estudo de usuários. Le Coadic (2004, p. 19) define assim a Ciência da Informação:

Preocupada em esclarecer um problema social concreto, o da informação, e voltada para o ser social que procura informação, situa-se no campo das ciências sociais 
(das ciências do homem e da sociedade), que são o meio principal de acesso a uma compreensão do social e do cultural.

Analisando-se a citação de Le Coadic, podemos concluir que a Ciência da Informação, ao participar do processo social, assume um papel importante quando, por meio das unidades de informação (UI), disponibiliza instrumentos, que são os produtos e serviços, de forma a possibilitar o acesso às informações pelos diversos usuários, tornando-se um agente transformador da vida dessas pessoas.

A esse respeito, Guinchat e Menou (1994, p. 486) afirmam que:

\begin{abstract}
A unidade de informação deve fazer todo o possível para conhecer bem as necessidades reais dos seus usuários e sua evolução, determinar o seu grau de satisfação e adaptar-se de acordo com isso. Além de estudos das necessidades e dos comportamentos, isto implica um contato pessoal tão estreito quanto possível com o usuário. Suas críticas, conselhos e sugestões devem ser solicitados e ouvidos.
\end{abstract}

A tarefa de disponibilizar informações adequadas a seus usuários requer a necessidade da identificação de suas demandas informacionais por meio dos estudos de usuários. No caso dos usuários com deficiência visual, o compromisso do profissional da informação e o valor do seu trabalho são essenciais pelas dificuldades enfrentadas por esses usuários, pois na sua quase totalidade as informações não são adaptadas às suas necessidades especiais.

As bibliotecas, portanto, são responsáveis por facilitar, por meio dos PSI ofertados, o acesso e a aquisição de informações, não somente no formato impresso, mas também em gravações de áudio e em meio digital. São responsáveis, ainda, pela produção desses produtos por meio da adaptação dos materiais alternativos. Assim, a participação das bibliotecas no processo de inclusão social traz autonomia, permitindo que a pessoa tenha a liberdade de escolha das informações de que necessita.

Nesse contexto, é importante o conhecimento de como foi o histórico da aquisição de informação pelas pessoas com deficiência visual, para que se entenda a relevância dos produtos e serviços disponibilizados pelas bibliotecas, assunto a ser desenvolvido a seguir.

\title{
4. PRODUTOS E SERVIÇOS E O ACESSO À INFORMAÇÃo
}

$\mathrm{O}$ acesso à memória coletiva oral pelo ser humano iniciou-se, segundo McGarry (1999), desde os primórdios da humanidade, seguido do acesso à memória coletiva escrita em 1700 a.C. Para os deficientes visuais esse acesso se deu somente com a invenção da escrita braile em 1824, ou seja, a diferença no acesso entre os videntes (pessoas que enxergam) e os deficientes visuais é de cerca de 3500 anos, ocasionando, de acordo com Passos (2010, p. 52), um abismo intelectual, com defasagem no acesso. No Quadro 3, podemos visualizar a diferença no acesso à informação pelos dois sujeitos: 
QUADRO 3: Diferença do acesso à memória coletiva entre deficientes visuais e videntes

\begin{tabular}{|c|c|c|c|c|}
\hline Sujeitos & $\begin{array}{l}\text { Acesso } \\
\text { memória } \\
\text { coletiva oral }\end{array}$ & $\begin{array}{l}\text { Acesso à } \\
\text { memória } \\
\text { coletiva escrita }\end{array}$ & $\begin{array}{l}\text { Acesso } \\
\text { memória } \\
\text { coletiva } \\
\text { impressa }\end{array}$ & $\begin{array}{l}\text { Acesso à } \\
\text { memória } \\
\text { coletiva } \\
\text { digital }\end{array}$ \\
\hline $\begin{array}{l}\text { Videntes (que } \\
\text { enxergam } \\
\text { normalmente) } \\
\text { (1) }\end{array}$ & $\begin{array}{ll}\text { Desde } & \text { os } \\
\text { primórdios } & \text { da } \\
\text { humanidade } & \end{array}$ & $\begin{array}{l}1700 \text { a.C. } \\
\text { (MCGARRY) }\end{array}$ & $\begin{array}{l}\text { 1450: } \\
\text { surgimento da } \\
\text { imprensa de } \\
\text { Gutemberg } \\
\text { (MCGARRY) }\end{array}$ & $\begin{array}{l}\text { 1981: } \\
\text { primeiro PC } \\
(\mathrm{COADIC})\end{array}$ \\
\hline $\begin{array}{l}\text { Deficientes } \\
\text { visuais (2) }\end{array}$ & $\begin{array}{ll}\text { Desde } & \text { os } \\
\text { primórdios } & \text { da } \\
\text { humanidade } & \end{array}$ & $\begin{array}{l}\text { 1824: } \\
\text { surgimento da } \\
\text { escrita Braille }\end{array}$ & $\begin{array}{l}\text { 1893: } \\
\text { surgimento da } \\
\text { primeira } \\
\text { impressora de } \\
\text { Braille } \\
\text { (BROWN) }\end{array}$ & $\begin{array}{l}\text { 1983: } \\
\text { primeira } \\
\text { tecnologia } \\
\text { assistiva } \\
\text { (WALLING) }\end{array}$ \\
\hline $\begin{array}{lr}\text { Diferença } & \text { de } \\
\text { anos } & \text { na } \\
\text { possibilidade } & \text { de } \\
\text { acesso } & \text { aos } \\
\text { registros } & \text { entre } \\
(1) \text { e (2) } & \end{array}$ & Não há & $\begin{array}{l}\text { Cerca de } 3500 \\
\text { anos }\end{array}$ & $\begin{array}{lll}\text { Cerca } & \text { de } & 440 \\
\text { anos } & & \end{array}$ & $\begin{array}{l}\text { Cerca de dois } \\
\text { anos }\end{array}$ \\
\hline
\end{tabular}

Fonte: Passos (2010, p. 52).

Com o registro escrito de conhecimentos disponíveis em diversos suportes, a linguagem natural, que era a base da construção da memória coletiva, deixou de ser exclusiva. Surge então a necessidade de organização desses conhecimentos e, com isso, um local onde fossem armazenados os materiais. São criadas as bibliotecas e, mais tarde, a oferta de produtos e serviços para os seus usuários.

A redução do abismo intelectual depende, entre outros fatores, do papel da biblioteca e dos profissionais da informação como produtoras de materiais alternativos e facilitadoras de seu acesso. Para isso é necessário conhecer com mais profundidade o novo usuário da biblioteca e o que ele precisa em termos de informação. Nesse processo é fundamental saber, a princípio, o seu nível de visão, para decidir se essa informação deverá ser ofertada no formato impresso, em braile, ampliada, no formato em áudio, gravado pela voz humana ou em meio digital para ser lido pelos leitores de tela pela voz sintetizada.

Podemos considerar, segundo Veiga (1946), que os primeiros produtos de informação foram os materiais produzidos em braile. A escrita braile se iniciou com o reglete e a punção manuais, ferramentas para a sua produção. Na década de 1930, surgiram as máquinas braile mecânicas e elétricas (notadamente da empresa americana Perkins) até hoje utilizadas para registrar informações. Nessa época são criadas as primeiras imprensas braile, equipamentos

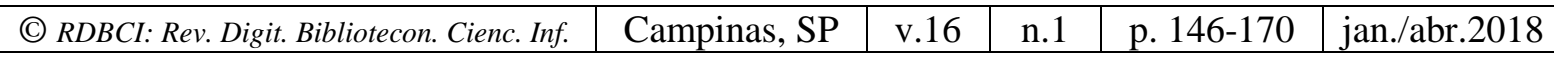


de grande porte para produção de braile em larga escala.Ao final do século XVII, segundo o autor, veio a ideia de se fixar os tipos de imprensa em hastes arrumadas de modo que os cegos pudessem escrever, batendo com essas hastes.

Como as imprensas e máquinas braile só produziam letras e textos lineares, foi criada uma máquina importante, o Termoform, um dispositivo utilizado para fazer desenhos em braile, como mapas e gráficos. Mais tarde surgiram os gravadores (os antigos com fitas magnéticas, os portáteis com fitas cassete, os digitais) que permitiam a captação, o transporte e o armazenamento de dados, como suporte para a informação em áudio, muito importante para as pessoas com deficiência visual.

No Brasil, na década de 1950, foi criada a Fundação para o Livro do Cego, que passou a se chamar Fundação Dorina Nowill, a primeira imprensa braile queatua nas diversas áreas da deficiência visual. Em 1975, essa fundação inicia um trabalho de capacitação de cegos, no uso de um aparelho chamado Optacom, que permitia aos cegos tomar contato com a forma escrita percebendo o contorno das imagens.

Na década de 1970, surgem aparelhos mais sofisticados, como o programa LIBRA (Listador braile), desenvolvido por programadores com deficiência visual da Companhia de Processamento de Dados do Município de São Paulo. Por meio de adaptações em impressoras convencionais, o LIBRA realiza a impressão de caracteres braile, permitindo que esses programadores não precisem mais de ledores humanos para lhes ajudar na correção e finalização de seus programas.

Um acontecimento fundamental, no início da década de 1980, foi a criação do primeiro sintetizador de voz pela IBM, o Roscoe, que foi o início dos leitores de tela, que possibilitavam aos deficientes visuais a leitura em meio digital.

No final da década de 1980 surgiram os microcomputadores, os primeiros scanners, as primeiras impressoras braile, os softwares leitores de tela e sintetizadores de voz mais avançados.

Podemos considerar que o grande marco no acesso à informação pelas pessoas com deficiência visual foi a invenção da escrita braile. A partir daí e com a evolução de todos esses PSI apresentados, as formas de acesso à informação por esses usuários foram ampliadas.

\section{AS BIBLIOTECAS COMO MEIO DE INCLUSÃO SOCIAL}

O cenário do processo de inclusão social das pessoas com deficiência no Brasil tem evoluído e sido mais debatido. Trabalhos têm surgido cada vez mais na literatura da Ciência da Informação. Embora tendo uma legislação na área, há a necessidade de uma fiscalização

\begin{tabular}{|c|c|c|c|c|c|}
\hline (C) RDBCI: Rev. Digit. Bibliotecon. Cienc. Inf. & Campinas, SP & v.16 & n.1 & p. $146-170$ & jan./abr.2018 \\
\hline
\end{tabular}


mais eficaz para que as leis sejam cumpridas. Além desse cumprimento, é necessário um trabalho de conscientização da sociedade no que diz respeito à aceitação das diferenças. Devem fazer parte desse trabalho ações educativas que venham a preencher a falta de informações sobre a deficiência. Os dois movimentos são fundamentais nesse processo.

Como uma parte importante do processo de inclusão, o acesso à informação é fundamental. Em relação às pessoas com deficiência visual, que são maioria entre os deficientes no Brasil, no que diz respeito a esse acesso, as Unidades de Informação (UI) têm desenvolvido produtos e serviços, principalmente no meio digital, essenciais na leitura de documentos. Os produtos de tecnologia assistiva, em especial, têm sido um grande facilitador. Esses produtos são desenvolvidos pela tecnologia assistiva que é uma área do conhecimento, de característica interdisciplinar, que engloba produtos, recursos, metodologias, estratégias, práticas e serviços que dão mais autonomia, independência e qualidade de vida a pessoas com deficiência, incapacidades ou mobilidade reduzida (PORTAL BRASIL, 2010).

É indubitável a importância da informação como elemento de melhoria na qualidade de vida dos deficientes visuais, abrindo espaços para despertar importantes discussões tanto no âmbito político como no social.

No processo de inclusão as UI assumindo seu papel social como umas das responsáveis por facilitar o processo de aquisição de informações desses usuários, colaboram para evitar a marginalização dos deficientes visuais na sociedade e, em consequência, a exclusão de seus processos.

Os trabalhos para o usuário com deficiência visual foram iniciados pelas bibliotecas em épocas distintas nos diferentes países. Segundo Kavanagh e Skold (2009), em alguns países, os serviços de biblioteca para pessoas cegas e incapazes de ler materiais impressos iniciaram-se há mais de dois séculos. Mulheres filantrópicas nos Estados Unidos e na Europa fundaram serviços de caridade para deficientes e algumas dessas pessoas criaram serviços de bibliotecas para cegos. Com o objetivo de propagar o cristianismo, mulheres missionárias distribuíram bíblias em formato acessível e foram as responsáveis pela fundação de bibliotecas para cegos na Ásia e na África.

Além do mais, muitas bibliotecas para cegos surgiram a partir de serviços de reabilitação para veteranos de guerra com limitações visuais. Eram mais uma extensão dos serviços de reabilitação para pessoas cegas, agências de caridade e não faziam parte do sistema de bibliotecas nacional. 


\subsection{No Exterior}

Sendo os Estados Unidos referência em bibliotecas para deficientes visuais, apresentaremos um breve histórico sobre o tema. Segundo Gerstenberger (1985), a história da inclusão das pessoas com deficiência visual nas bibliotecas se deu da seguinte forma. Provavelmente o primeiro serviço de biblioteca para cegos nos Estados Unidos foi o empréstimo de livros em relevo (braile e vários outros métodos de escrita) pela Biblioteca Pública de Boston por volta de 1868. E o primeiro reconhecimento por parte do governo federal de que as pessoas cegas poderiam ler foi a ratificação, em 1904, da legislação que permitiu material de leitura em relevo para cegos para ser enviado grátis através do correio postal americano.

Segundo Farrel (2012), o processo de inclusão dos deficientes visuais foi iniciado quando John Russell Young, bibliotecário do Congresso em 1897, criou uma sala de leitura para cegos com cerca de 500 livros e itens de música. Na década de 1890, a Biblioteca Pública de Boston, a Biblioteca Livre de Filadélfia, a Biblioteca de Chicago, a Biblioteca de Circulação gratuita para Cegos de Nova York e a Biblioteca Pública de Detroit começaram a oferecer materiais para deficientes visuais. A Biblioteca do Estado de Nova York foi a primeira a criar um departamento destinado a esses usuários.

Em 1913, o parlamento americano exigiu que uma cópia de cada livro publicado para fins educacionais, sob o subsídio do governo pela American PrintingHouse for the Blind, fosse depositado na Biblioteca do Congresso. Lentamente, os materiais eram disponibilizados em formato acessível. Um acontecimento que se tornou um marco no acesso à informação pelos deficientes visuais foi quando o Presidente Hoover assinou a Lei Pratt-Smoot em 1931, dando apoio mais amplo para os serviços aos deficientes visuais. Esta lei autorizou uma verba anual de US \$ 100.000 pela Biblioteca do Congresso para fornecer livros para residentes adultos cegos nos Estados Unidos e seus territórios.

O programa evoluiu para o Serviço de Bibliotecas Nacionais para deficientes visuais e físicos. A criação de um sistema uniforme de Braille em inglês e o surgimento do livro falado, em 1933, mostrou uma melhoria nesses serviços. Em 1952, a legislação foi ampliada para incluir serviços para crianças cegas. Dez anos depois, o programa foi acrescido de uma biblioteca de partituras musicais e texto instrutivo para cegos e, em 1966, o Projeto de Lei n. 889-522 autorizou a criação de livros falados para todas as pessoas que não pudessem ler material impresso padrão por deficiência física ou visual.

Ainda permanecem barreiras e, em 1996, o senador Chaffee apresentou uma emenda à Lei de Direitos Autorais, que eliminou a necessidade de agências governamentais e sem fins lucrativos pedirem a permissão de editores ou proprietários de direitos autorais para reproduzir materiais impressos em formatos especiais para leitores cegos ou fisicamente 
incapacitados (NLS, 1983). De um começo de 19 bibliotecas cooperantes, a rede se expandiu para 57 regionais e 86 bibliotecas sub-regionais.

A Biblioteca do Congresso, as grandes bibliotecas urbanas e as bibliotecas estaduais americanas têm desempenhado um papel fundamental no desenvolvimento das bibliotecas para deficientes visuais. Atualmente, o National Library Service for the Blind and PhysicallyHandicapped (NLS), da Library of Congress, administra o Serviço de Livros Falados e em braile, um programa gratuito no qual são feitos empréstimos dos livros gravados, livros e revistas em braile, partituras musicais em braile e em letras ampliadas, e também dos equipamento de reprodução para os residentes dos Estados Unidos que são incapazes de ler ou utilizar materiais impressos padrão, por causa de deficiência visual ou física. Esse material é enviado pelas bibliotecas cooperantes locais, que remetem pelo correio todo esse material, diretamente aos inscritos sem nenhum custo. Livros falados, revistas e publicações em braile são entregues aos usuários por correio livre de postagem e através de uma rede de bibliotecas cooperativas (WILSON, 2003).

A Dinamarca e a Suécia seguem o modelo americano no qual uma rede nacional de bibliotecas para cegos é totalmente custeada pelo governo. Já no Canadá, Austrália e Reino Unido os serviços para as pessoas com deficiência visual desenvolveram-se fora do sistema de bibliotecas principal, mais como uma extensão das agências de caridade às quais se comprometiam com a sua reabilitação e o fornecimento de livros adaptados às suas necessidades de leitura (KAVANAGH; SKOLD, 2009).

O Canadá, com o programa Visunet Canada PartnersProgramme, uma parceria entre as bibliotecas públicas e serviços de bibliotecas para cegos, seguiu o caminho da cooperação. Esse modelo não é baseado somente na cooperação, mas também na integração do serviço para cegos com os serviços convencionais da biblioteca. Por meio desse programa, a biblioteca local tem acesso a um catálogo online da coleção em vários formatos e a um número de recursos digitais incluindo jornais, revistas e as publicações disponíveis em formato eletrônico (GRIEBEL, 2000).

A Biblioteca Nacional da Austrália, tem o papel de agente facilitador na coordenação e cooperação entre os serviços de bibliotecas que disponibilizam PSI para os usuários com as diversas deficiências. Realiza esse trabalho por meio de uma seção da biblioteca criada para este fim, disponibilizando e mantendo um catálogo nacional de materiais em formato alternativo, o Catálogo Nacional de Materiais para Pessoas com Deficiência (NUC: D) ofertados pelas bibliotecas e produzidos em toda a Austrália (AUSTRALIAN LIBRARY AND INFORMATION ASSOCIATION, 1998).

No Reino Unido a National Library for the Blind (NLB) foi uma biblioteca pública, fundada em 1882, que visava garantir que as pessoas com deficiência visual tivessem o mesmo acesso aos serviços de biblioteca que os demais usuários. Em 2007, o NLB foi 
incorporado pelo Royal National Institute of Blind People (RNIB), uma instituição de caridade, e passou a fazer parte do Serviço de Bibliotecas Nacionais do RNIB (ROYAL NATIONAL INSTITUTE OF BLIND PEOPLE, 2017).

\subsection{No Brasil}

A realidade brasileira se encontra em um momento diferente do exterior, assim como o processo de inclusão social das pessoas com deficiência.

No que diz respeito à educação, os deficientes começaram a ganhar visibilidade para a sociedade brasileira, na década de 1950, quando a política educacional brasileira criou a educação especial, com classes especiais e, dessa forma, iniciou a inclusão dos deficientes por meio da educação. Já a educação dos deficientes visuais teve seu início com a fundação do Imperial Instituto dos Meninos Cegos, no Rio de Janeiro, em 1891, mais tarde denominado Instituto Benjamin Constant. Destinava-se ao ensino primário e alguns ramos do secundário, oferecendo educação moral e religiosa, música, ofícios fabris e trabalhos manuais (JANNUZZI, 2006). Posteriormente o Instituto criou uma biblioteca com materiais em braile.

Para se entender como foi o início do surgimento de serviços de bibliotecas para deficientes visuais, será mencionado um breve histórico.

O registro mais antigo encontrado sobre uma biblioteca para cegos é no momento da fundação da Associação Promotora de Instrução e Trabalho para os Cegos (APIT), na cidade de São Paulo, quando no seu manifesto de lançamento assinado por 14 cegos consta a criação de uma biblioteca. A APIT é atualmente ativa, mas a ideia da biblioteca não foi adiante (GIL, 2012).

Segundo Masine (2014), em 1943 foi instalada na Escola Caetano de Campos, na cidade de São Paulo, uma Biblioteca Braille. Dorina de Gouveia Nowill foi convidada a se matricular como aluna regular dessa escola. A partir de uma visita ao Instituto Padre Chico para Cegos, Dorina e um grupo de colegas aprenderam o sistema braile e criaram cartilhas e livros de leitura intermediária. Nesse momento foi deflagrada uma mudança na educação. Dorina, percebendo, naquela época, a carência de livros feitos em braile no Brasil, criou, com outras normalistas, a Fundação Dorina Nowill para Cegos.

Em 1946, foi também criado o Setor de Livros em Braile da Biblioteca Infantil Monteiro Lobato, na capital paulista, que logo se transformou em sala para que alunos com deficiência visual passassem a frequentá-la. Inicia-se aí o primeiro serviço para cegos em uma biblioteca pública no Brasil. Em 1947, foi oficializada a primeira experiência de educação de cegos com a criação do primeiro curso da América Latina de formação de professores de educação de cegos, na Escola Caetano de Campos (NOWILL, 2002). 
A partir daí, começaram a surgir serviços de biblioteca para os usuários com deficiência visual e foram sendo disponibilizados pequenos acervos em braile chamados de Setores Braille. As bibliotecas criadas especificamente para esses usuários são poucas e em geral, esses serviços são concentrados no serviço de referência das bibliotecas.

Vale ressaltar o papel das bibliotecas das universidades brasileiras públicas e privadas, que têm demonstrado a preocupação em ofertar esses PSI, como o Laboratório de Acessibilidade da Universidade Estadual de Campinas (UNICAMP), a Biblioteca da Universidade Federal do Rio Grande do Norte, a Biblioteca Central da Universidade de Brasília (UnB), a Biblioteca da Universidade Estadual de Londrina (UEL), entre outras (MALHEIROS, 2013, p. 28).

Essas bibliotecas investem no processo de inclusão desses usuários, cumprindo uma função importante no auxílio à permanência desses alunos e fornecendo a informação adaptada e necessária a seu desenvolvimento, tendo implementado diversas ações para facilitar a permanência desses alunos e diminuir suas dificuldades por meio de seus órgãos de inclusão, dando apoio à vida universitária (idem, 2013, p. 28).

Também as bibliotecas privadas têm ofertado PSI para os usuários com deficiência visual e como exemplo podemos citar as bibliotecas da Fundação Dorina Nowill e do Instituto Benjamin Constant, que são as maiores bibliotecas brasileiras destinadas aos usuários com deficiência visual.

Somadas a essas bibliotecas estão as bibliotecas digitais acessíveis, que pelas facilidades trazidas pela informação em meio digital para os usuários em questão, estão crescendo a cada dia. Como exemplo podemos citar, a Biblioteca Digital para Deficientes Visuais da Universidade Metodista de São Paulo, a Biblioteca Virtual Sonora da Universidade Estácio de Sá de Campos dos Goytacazes, a Biblioteca Digital da Fundação Dorina Nowill, a Dorinateca, a Biblioteca Digital Acessível, do Ministério da Educação (MEC), a Biblioteca Digital da Universidade Estadual de Campinas (UNICAMP), e a Biblioteca Digital e Sonora (BDS), da Universidade de Brasília (UnB), o Repositório de Informação Acessível da Universidade Federal do Rio Grande do Norte (RIA/UFRN), Biblioteca Virtual da Associação Catarinense para a Integração do Cego (ACIC), entre outras (MALHEIROS, 2013, p. 35, SANTOS; ARAÚJO, 2015).

\section{OS PRODUTOS E SERVIÇOS DE INFORMAÇÃO}

Nas bibliotecas, o setor de referência é a área-chave, sendo responsável pela disponibilização dos produtos ofertados, os quais têm se desenvolvido cada vez mais no ambiente da internet. A disponibilização de serviços por meio da chamada Web 2.0 resultou em mais agilidade e eficiência no atendimento aos usuários. 
Os produtos das bibliotecas e sua divulgação disponibilizados pelo serviço de referência devem ser construídos dentro do conceito universal para alcançar todos os usuários. Se a equipe que dirige a biblioteca tem uma visão inclusiva, as atividades serão direcionadas nesse sentido e o produto será também inclusivo atendendo a todos os usuários. Assim como os serviços de referência para os usuários em geral, aqueles serviços destinados aos usuários com deficiência visual também caminham para os recursos em meio digital.

Como exemplo podemos citar o Serviço de Referência da Northern Illinois University'sFounders Memorial Library, analisado por Tinerella e Dick (2005), que também mostraram outros serviços de referência de bibliotecas universitárias para usuários com deficiência visual. Esse serviço geralmente conta com o auxílio de um estudante com deficiência visual treinado para o atendimento. O treinamento foi iniciado na década de 1990 e com essa atitude da biblioteca os alunos ganharam experiência e referências de trabalho. Essa ação faz parte do programa University Libraries Services for Personswith Disabilities Program, desenvolvido no início dos anos 1980.

Os PSI disponibilizados pela biblioteca e apontados por esses dois autores são:

- Dispositivos eletro-ópticos, como o reconhecimento de caracteres óticos e utilitários para a ampliação de tela; software de ampliação; circuito fechado de televisão; processadores de imagens visuais; scanners; sistemas de leitura para alunos com baixa visão; dispositivos adaptados de comunicação escrita, tais como teclados ampliados e máquinas de escrever.

- Dispositivos de substituição sensorial, como livros falados, calculadoras, gravações em áudio, sintetizadores de voz, compressores de fala, marcadores auditivos e máquinas de leitura; leitores em braile, máquinas de escrever em braile, teclados e monitores; dispositivos de tamanho relativo que ampliam material impresso, símbolos e outros itens.

- Dispositivos ópticos para pessoas com baixa visão, incluindo óculos, ampliadores de mãos e pés, de lentes UV telescópicas e absortivas; dispositivos de iluminação, brilho, contraste e cor; e livros ampliados e revistas.

Uma atmosfera agradável deve ser oferecida a esses alunos para que desperte um sentimento de confiança de que serão atendidos quando precisarem. As bibliotecas universitárias podem, com algumas acomodações a um baixo custo, dar um bom atendimento a seus usuários com deficiência visual.

Os autores concluem que os alunos com deficiência frequentemente se sentem isolados e são sensíveis à percepção de que são diferentes. Com uma atitude positiva, as bibliotecas das universidades podem fazê-los se sentir acolhidos. E se "a biblioteca acadêmica está verdadeiramente comprometida com a plena inclusão e igualdade de oportunidades, então a participação e o comprometimento de todo o pessoal de referência é essencial" (TINERELLA; DICK, p. 32, 2005, tradução nossa). 
Para Monteiro (2010), são inúmeras as possibilidades que o serviço de referência e informações têm por meio da tecnologia assistiva de atender aos usuários com deficiência visual e do seu papel de mediador da questão do usuário ao ambiente da unidade de informação. Esse serviço sofreu mudanças com a chegada da informação digital e, atualmente, de forma crescente, tarefas são realizadas no computador. São tarefas desse serviço: fazer a mediação entre a informação e o usuário; auxiliar na busca, recuperação e no acesso a informações e documentos; propiciar a educação para o uso desses recursos e do sistema; fazer o trabalho de alerta e disseminação seletiva da informação; divulgar novos produtos e serviços; e realizar o planejamento e a supervisão dessas atividades direcionadas para esse público.

Como afirma o autor acima citado, a tecnologia assistiva (TA) trouxe muitas possibilidades para os serviços de referência no atendimento aos usuários com deficiência visual. Os recursos de TA não só facilitaram como possibilitaram o acesso à informação por esses usuários. A informação em meio digital abriu novos horizontes de acesso e a TA contribuiu para a sua concretização.

Bernardi (2004), em sua revisão de literatura, lista os serviços fornecidos pelas agências nacionais ou locais e pelos projetos de redes de bibliotecas que são: materiais especiais em formato tradicional, como braile, livros falados em áudio e livros ampliados; tecnologia assistiva, acompanhadas de treinamento para os usuários e bibliotecários; livros em áudio, CD-ROM, braile e livros ampliados ocupam uma parte crescente dos serviços das bibliotecas públicas; serviços tais como o acesso aos catálogos específicos, textos digitais, livros falados digitais, livros no formato Daisy e um formato especial de empréstimo entre bibliotecas.

Assim, como podemos observar, entre as diversas possibilidades de produtos e serviços, destaca-se a tecnologia assistiva (TA), por meio da qual esses usuários acessam programas de computador, internet e recursos digitais usando o braile, ampliação de tela, software de digitalização com OCR, leitores de tela e síntese de fala (idem, 2004).

Para acessar informações, os usuários com deficiência visual necessitam de recursos diferentes dependendo do seu resíduo de visão. Isso se aplica tanto ao meio impresso como ao meio digital. Sonza (2008) divide a TA em dois grupos: interfaces para usuários com baixa visão e interfaces para usuários cegos, levando em consideração que as necessidades de cada grupo são distintas. Para os usuários cegos os recursos de software são os leitores de tela ou sintetizadores de voz, que são programas que identificam e interpretam as informações exibidas na tela do monitor e repassam essas informações por meio de síntese de voz, e que estabelecem um diálogo com o usuário por meio de aplicativos próprios, com voz humana gravada. Também os usuários com baixa visão severa podem se utilizar desses recursos. As interfaces para os usuários com baixa visão leve e moderada, que têm um resíduo maior de visão, podem ser a ampliação de tela e o contraste. 
Em relação ao hardware para os usuários cegos as interfaces são as impressoras braile, que imprimem material em braile e que podem ter a função de impressão acoplada a outras funções. Podem vir com o Programas Duxbury, com o programa Braille Fácil e a impressora em relevo, o Thermoform, que copia material adaptado em alto relevo para que os usuários possam utilizar gráficos, mapas, desenhos. Outros recursos de hardware são os dispositivos de saída em braile, o braile falado, que é um sistema portátil de armazenamento e processamento da informação, no qual a entrada dos dados é feita através de um teclado braile de seis pontos e a saída é feita por meio de um sintetizador de voz; o terminal braile (linha braile), equipamento eletrônico ligado ao computador e o braile lite, assistente pessoal que funciona como um Palm pilot, com um caderno para tomar notas, um calendário e uma agenda (SONZA, 2008).

Existem hoje, no mercado mundial, diferentes tipos de impressoras Braille, seja para uso individual (pequeno porte) ou para produção em larga escala (médio e grande porte). As velocidades de produção são muito variadas. Essas impressoras, geralmente, podem imprimir Braille interpontado ou não em seis ou oito pontos, bem como produzir desenhos. Algumas impressoras Braille podem utilizar folha solta, mas a maioria funciona com formulário contínuo (CERQUEIRA; FERREIRA, 2000).

A impressora em relevo, ou thermoform utiliza para impressão a folha de plástico (GermanPaper), para desenho (bidimensional). É utilizada para a impressão de gráficos, mapas, etc. Trata-se de um sistema de moldagem por vácuo de uma película plástica aquecida, que é desse modo comprimida contra um molde (a preparação deste molde pode ser efetuada por processos informatizados ou artesanais). Também pode ser usado na confecção de cópias de textos braile (A HISTÓRIA..., 2008).

Os leitores de tela ou sintetizadores de voz são muito utilizados por usuários cegos e também por usuários com baixa visão severa, na leitura de textos em meio digital. São vários os leitores atualmente disponíveis: o Dosvox,o Virtual Vision, o Jaws, o Non Visual Desktop Access (NVDA) (para ambiente Windows), o Orca (para Linux), o Voice Over (para Mac OS) e outros.

Uma pesquisa importante sobre o uso dos leitores de tela por pessoas com deficiência foi realizada em 2015, pela organização Webaim - Center for Personswith Disabilities, da Utah StateUniversity. Os leitores de tela mais utilizados foram: Jaws, 30.2\%; ZoomText, 22.2\%; Window-Eyes, 20.7\%; NVDA, 14.6\%; VoiceOver, 7.6\%; System Access or System Access To Go, 1.5\%; ChromeVox, 0.3\%. Segundo a pesquisa, a tendência de janeiro de 2014 a julho de 2015 é a caída do Jaws e NVDA, a subida do Zoom text e do Windows-Eyes, e o Voiceover e o System acessto go se mantiveram estáveis (SCREEN..., 2015).

Malheiros (2009), de acordo com o resultado de seu estudo do usuário deficiente visual da Universidade de Brasília, constata que a informação em meio digital é a mais utilizada por 
esses usuários. Por esse motivo, é muito importante que os profissionais responsáveis por desenvolver essas interfaces estejam atentos à questão do desenho universal, que, segundo Sonza (2008), tem o objetivo de valorizar a diversidade humana, para evitar a situação de exclusão. A desatenção a essa questão tem como consequência a limitação de acesso a um mundo de informações que estão disponíveis na internet.

\section{CONSIDERAÇÕES FINAIS}

Os PSI ofertados pelas bibliotecas são um ponto fundamental na autonomia das pessoas com deficiência visual e, para desenvolvê-los, é necessário a sua inclusão no planejamento geral da biblioteca. É necessária a atenção ao desenvolvimento de coleções por meio de um estudo de usuários, com o objetivo de prestar um bom atendimento às necessidades de informação para os usuários com deficiência visual, com isso reduzindo os custos do processo de produção de materiais alternativos e tendo como resultado PSI mais eficientes.

Os estudos de usuários com deficiência visual são insuficientes e esse usuário geralmente é negligenciado no planejamento das bibliotecas brasileiras. Esses estudos são essenciais no desenvolvimento da coleção de qualquer biblioteca e principalmente em relação ao usuários com deficiência visual que têm particularidades como o nível de visão, que é um fator determinante para a oferta de produtos e de serviços, vão garantir uma coleção criada de acordo com o que a comunidade em questão precisa em termos de informação (RABELO, 1989; FIGUEIREDO, 1994; CASELLI, 2007; MALHEIROS, (2009, 2013); RADOS, VARVAKIS e BLATTMANN, 1999; MARQUEZ e DOWNEY, 2016).

Uma questão importante e que talvez seja a solução para as questões apresentadas é a cooperação entre bibliotecas como meio de oferecer um serviço de melhor qualidade. A questão da cooperação, das parcerias e da descentralização dos serviços de bibliotecas para cegos e pessoas que não leem material impresso padrão são elementos que vão equalizar esses serviços. (RABELO (1989); GRIEBEL (2000); BERNARDI (2004); HIRSCHFELDT, 2005; KAVANAGH; SKOLD $(2005,2009))$.

O futuro dos serviços de bibliotecas para deficientes visuais segue dois modelos que estão surgindo nas bibliotecas públicas e especializadas. Um é o modelo híbrido de biblioteca que integra os serviços tradicionais e os eletrônicos, que é o caso do Reino Unido. Seria então um "serviço híbrido". O outro modelo é altamente digital e tecnológico, que é a tendência nos Estados Unidos. As tendências gerais desses serviços vão no sentido de políticas de serviços compartilhados entre as bibliotecas para cegos, agências e bibliotecas, caminhando em direção à tecnologia digital (BERNARDI, 2004).

A criação de uma rede cooperativa de bibliotecas para usuários com deficiência visual é apontada pelos autores acima citados como solução na diminuição dos custos de produção, 
evitando a duplicação de trabalho e disponibilizando um número maior de informações. Cooperação, integração e compartilhamento são as palavras chave para a resolução da questão do acesso à informação pelas pessoas com deficiência visual.

\section{REFERÊNCIAS}

A HISTÓRIA da leitura para pessoas com deficiência. 2008. Disponível em: $<$ http://www.livroacessivel.org/a-leitura-e-as-pessoas-com-deficienciavisual.php\#conteudo>. Acesso em: 19 out. 2016.

\section{AUSTRALIAN LIBRARY AND INFORMATION ASSOCIATION. Guidelines on library} standards for people with disabilities. 1998. Disponível em: https://www.alia.org.au/aboutalia/policies-and-guidelines/alia-policies/guidelines-library-standards-people-disabilities. Acesso em: 3 set. 2017.

BEAL, A. Gestão estratégica da informação: como transformar a informação e a tecnologia da informação em fatores de crescimento e de alto desempenho nas organizações. São Paulo: Atlas, 2004.

BERNARDI, F. Library services for blind and visually impaired peoples. 2004. Trabalho de Conclusão de Curso (Especialização) - Università degli Studi, Parma, 2004. Disponível em: <http://dspaceunipr.cineca.it/bitstream/1889/1147/1/Library\%20Services\%20for\%20Blind\%20and\%20Visu ally\%20Impaired\%20People\%20A\%20Literature\%20Review.pdf >. Acesso em: 19 jun. 2016.

BRAZIER, H. Sharing the Vision. CILIP UPDATE with gazette, London, p. 40-42,Jul. 2011.

BROWN, R. A history for publishing for people who are blind. History Buff.com-A nonprofitOrganization.2012. Disponível em:

<http://www.historybuff.com/library/refbraille.html>. Acesso em: 12 jun. 2017.

BUCKLAND, M. Information as thing. Journal of American Society for Information Science, v. 45, n. 5, p. 351-360, 1991.

CASELLI, B. Acesso à informação digital por portadores de necessidades especiais visuais: estudo de caso telecentro acessível de Taguatinga. 2007. 94 f. Dissertação (Mestrado em Ciência da Informação) - Programa de Pós-Graduação em Ciência da Informação e Documentação, Universidade de Brasília, Brasília, 2007.

CERQUEIRA, Jonir Bechara;FERREIRA, Elise de Melo Borba. Recursos didáticos na educação especial. Disponível em:

<http://www.ibc.gov.br/images/conteudo/revistas/benjamin_constant/2000/edicao-15abril/Nossos_Meios_RBC_RevAbr2000_ARTIGO3.pdf>. Acesso em: 8 ago. 2017. 
DAVIES, J. E. An overview of international research into the library and information needs of visually impaired people. Library Trends, v. 55, n. 4, p. 785-795, 2007.

DUARTE, E. J. et al. Os serviços e os produtos de informação oferecidos pela biblioteca pública de Santa Catarina. Revista ACB: Biblioteconomia em Santa Catarina, v. 20, n. 3, p. 606-620, set. /dez. 2015.

FARRELL, M.A Brief History of National Support for Libraries in the United States. In: WORLD LIBRARY AND INFORMATION CONGRESS, IFLA GENERAL CONFERENCE AND ASSEMBLY, 78., 2012. Helsinki, 2012. 10 p. Disponível em: https://www.ifla.org/past-wlic/2012/140-farrell-en.pdf. Acesso em: 27 ago. 2017.

FIGUEIREDO, N. M. Estudo de uso e usuários da informação. Brasília: IBICT, 1994.

FULLMER, S.; MAJUMBER, R. K. Increased access and use of disability related information for consumers. Journal of Rehabilitation, v. 57, p. 17-22, July/Sept. 1991.

GERBER, E. The Benefits of and barriers of computer use for individuals who are visually impaired. Journal of Visual Impairment and Blindness, p. 536-550, Sept. 2003.

GERSTENBERGER, D. Library services for the blind a brief review and overview. Future Reflections, v. 4, n. 2, Apr./May/June 1985.

GIL, Marta. Caminhos dainclusão: a história da formação profissional de pessoas com deficiência no Senai. São Paulo: Senai-SP, 2012. 246 p. Disponível em:

https://books.google.com.br. Acesso em: 4 set. 2017.

GRIEBEL, R. Partnering Services between Public Libraries and Library Services for the Blind: A Canadian experience. In: IFLA COUNCIL AND GENERAL CONFERENCE, 66. 2000. Conference proceedings. Jerusalem, 2000. 7 p. Disponível em: http://files.eric.ed.gov/fulltext/ED450764.pdf. Acesso em: 25 ag. 2017.

GUINCHAT, C.; MENOU, M. Introdução geral às ciências e técnicas da informação e documentação. 2. ed. Brasília: IBICT, 1994.

HIRSCHFELDT, I. B. Libraries for all: the Swedish way. In: WORLD LIBRARY AND INFORMATION CONGRESS, IFLA GENERAL CONFERENCE AND COUNCIL, 71., 2005. Oslo, 2005. 5 p.

JANNUZZI, Gilberta de Martino. A educação do deficiente no Brasil: dos primórdios ao início do século XXI. 2. ed. Campinas: Autores Associados, 2006. 
KAVANAGH, R.; SKÖLD, B. C. (Ed.). Bibliotecas para cegos na era da informação: diretrizes de desenvolvimento. São Paulo: Imprensa Oficial do Estado de São Paulo, 2009. (Relatório Profissional, 86).

KAVANAGH, R.; SKOLD, B. C. (Ed.). Libraries for the blind in the Information age: guidelines for development. The Hague: IFLA, 2005. (IFLA professional reports, 86). Disponível em: <http://www.ifla.org/files/assets/hq/publications/professional-report/86.pdf>. Acesso em: 8 out. 2016.

LE COADIC, Y. F. A ciência da informação. 2. ed. Brasília: Briquet de Lemos, 2004.

MACHELL, J. Library and information services for visually impaired people: national guidelines. London: Library Association, 1996.

MALHEIROS, T. M. C. Estudo do usuário deficiente visual e subsídios para uma política de desenvolvimento de coleções da Biblioteca Central da Universidade de Brasília. 2009. 94 f. Monografia (Especialização em Gestão Universitária) - Universidade de Brasília, Brasília, 2009.

MALHEIROS, T. M. C. Necessidade de informação do usuário com deficiência visual: um estudo de caso da Biblioteca Digital e Sonora da Universidade de Brasília. 2013. 305 p. il. Dissertação (Mestrado em Ciência da Informação) - Universidade de Brasília, Brasília, 2013.

MARQUEZ, J. J.; DOWNEY, A. Library Service Design: A LITA Guide to Holistic Assessment, Insight, and Improvement (LITA Guides).Lanham, MD:

Rowman\&LittlefieldPublishers, 2016. 144 p.

MASINI, E. F. S. O perceber de quem está na escola sem dispor da visão. São Paulo: Cortez, 2014.

MCGARRY, K. O contexto dinâmico da informação: uma análise introdutória. Brasília: Briquet de Lemos, 1999.

MONTEIRO, E. C. Souza de Aguiar. O serviço de referência e a acessibilidade aos deficientes visuais. In: SEMINÂRIO NACIONAL DE BIBLIOTECAS UNIVERSITÁRIAS, 16., 2010, Rio de Janeiro; SEMINÁRIO NACIONAL DE BIBLIOTECAS DIGITAIS, 2. 2010, Rio de Janeiro. Anais... Rio de Janeiro: UFRJ, 2010.

NLS. That all may read: library services for blind and physically handicapped people. Washington DC: Library of Congress, 1983. Disponível em: <https://archive.org/stream/thatallmayreadli00libr/thatallmayreadli00libr_djvu.txt>. Acesso em: 18 out. 2016. 
NOWILL, D. G. Fundação Dorina Nowill para cegos. In: MASINI, E. F. S. (Org.). Do sentido, pelo sentido, para o sentido: sentido das pessoas com deficiência sensorial. Niteroi: Intertexto; São Paulo: Vetor, 2002.

OMVIG, J. History of blindness: summary of the history of the education and rehabilitation of the blind. 2016. Disponível em: <https://www.actionfund.org/history-blindness>. Acesso em: 15 out. 2016.

PASSOS, J. dos R. A informationliteracy e os deficientes visuais: um caminho para a autonomia? 2010. 174 f. Tese (Doutorado em Ciência da Informação) - Escola de Comunicação e Artes da Universidade de São Paulo, São Paulo, 2010.

PORTAL BRASIL. Tecnologia assistiva ajuda a melhorar a qualidade de vida de pessoas com deficiência. 2010. Disponível em: http://www.brasil.gov.br/sobre/ciencia-etecnologia/desenvolvimento-sustentavel/tecnologia-assistiva. Acesso em: 3 out. 2016.

RABELLO, O. C. P. O deficiente visual e a biblioteca pública estadual "Luiz de Bessa". Revista da Escola de Biblioteconomia da UFMG, v. 18, n. 1, p. 39-60, mar. 1989.

RADOS, G. J. V.; VALERIM, P.; BLATTMANN, Ú. Valor agregado a serviços e produtos de informação. Informativo CRB 14/ACB, v. 9, n. 1, p. 11-12, jan./mar. 1999. Disponível em: <http://www.reocities.com/ublattmann/papers/valor.html>. Acesso em: 16 abr. 2016.

RAPOSO, P. N. Manual de adaptação e descrição de figuras. Brasília: Universidade de Brasília, Laboratório de Apoio ao Deficiente visual (LDV), [200-?]. Manual nãopublicado.

ROYAL NATIONAL INSTITUTE OF BLIND PEOPLE. 2017. Who we are. Disponível em: http://www.rnib.org.uk/who-we-are. Acesso em: 3 set. 2017.

SANTOS, C. G.; ARAÚJO, W. J. Aspectos de vulnerabilidades em bibliotecas digitais acessíveis. Encontro Nacional de Pesquisa em Ciência da Informação, v. 16, 2015.

SCREEN readerusersurvey \#6 results. Web Accessibilityin Mind, 2015. Disponível em: <http://webaim.org/projects/screenreadersurvey6/\#disabilitytypes>. Acesso em: 8 nov. 2016.

SONZA, A. P2008. 298f. Tese (Doutorado em Informática na Educação)- Programa de PósGraduação em Informática na Educação, Universidade Federal do Rio Grande do Sul, Porto Alegre, 2008. Disponível em: <http://www.bento.ifrs.edu.br/ept/tese>. Acesso em: 5 ago. 2017.

TINERELLA, V. P.; DICK, M. A. Academic reference service for the visually impaired: a guide for the non-specialist. College\& Research Libraries News, v. 66, n. 1, p. 29-32, Jan. 2005 . 
VEIGA, J. E. A vida de quem não vê. Rio de Janeiro: J. Olympio, 1946. Disponível em: <http://www.deficienciavisual.pt/r-A_vida_de_quem_nao_ve-Espinola_Veiga.htm>. Acesso em: 14 out. 2016.

VITZANSKY, W. Managing a national library service for blind and print-handicapped persons. Library Management, v. 15, n. 7, p. 23-28, 1994.

WALLING, L. L. Disabilities, children and libraries: mainstreaming services in public libraries and school libraries media centers. Colorado: Libraries Unlimited, 1993, p. 43-45, 204-219.

WILSON, A. History of the service for the blind and physically handicapped. 2003. Disponível em: <http://www.michigan.gov/libraryofmichigan/0,2351,7-160-19270_2895879831--,00.html>. Acesso em: 15 out. 2016.

WORLD BLIND UNION. June 17 Press Release for WIPO Book Treaty. 2013.

Disponível em: http://www.worldblindunion.org/English/news/Pages/JUne-17-Press-Releasefor-WIPO-Book-Treaty.aspx. Acesso em: 25 ago. 2017.

WTBBL. History of the Washington talking book \& braille library. [201-?]. Disponível em: <http://www.wtbbl.org/History.aspx>. Acesso em: 17 out. 2016.
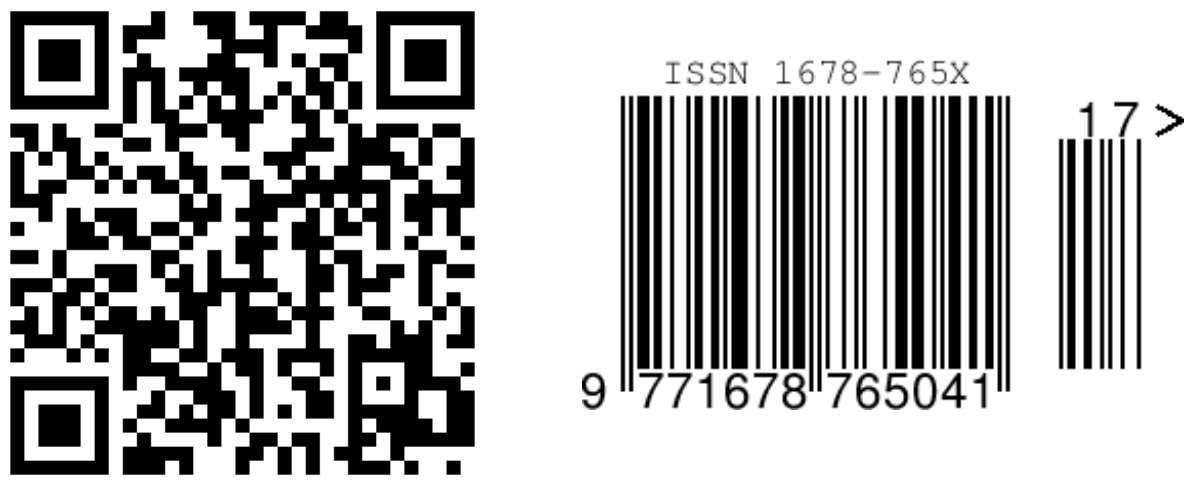